Die mít Sicherheit bis jetzt rekognoszierten basischen Bestandteile der weilsen Nieswurz wären sonach:

$\begin{array}{ll}\text { Protoveratrin: } & \mathrm{C}_{32} \mathrm{H}_{51} \mathrm{NO}_{11} \\ \text { Protoveratridin: } & \mathrm{C}_{26} \mathrm{H}_{45} \mathrm{NO}_{8} \\ \text { Pseudojervin: } & \mathrm{C}_{29} \mathrm{H}_{43} \mathrm{NO}_{7} \\ \text { Jervin: } & \mathrm{C}_{26} \mathrm{H}_{37} \mathrm{NO}_{3} \\ \text { Rubijervin: } & \mathrm{C}_{26} \mathrm{H}_{43} \mathrm{NO}_{2} .\end{array}$

\title{
Über das Kessoöl.
}

Von J. Bertram und E. Gildemeister.

(Eingegangen den 4. VIII. 1890.)

Die Wurzel der Valeriana officinalis varietas angustifolia, in ihrer Heimath Japan Kesso oder Kanokosô genannt, liefert bei der Destillation mit Wasserdämpfen etwa 8 Proz. eines ätherischen Öles, welches äufserlich, sowie im Geruch und Geschmack, ganz mit dem gewöhnlichen Baldrianöl übereinstimmt, jedoch durch sein höheres spez. Gewicht von diesem abweicht. Dasselbe beträgt beim Kessoöl etwa 0,996, während es beim gewöhnlichen Baldrianöl um 0,945 herum liegt.

Letzteres ist in der Wurzel der bei uns heimischen Valeriana officinalis zu etwa 1 Proz. enthalten und ist früher von Bruylants1 zum Gegenstand einer Untersuchung gemacht worden. Er fand in demselben ein bei 155 bis $160^{\circ}$ siedendes Terpen, welches mit Salzsăure eine krystallisierende Verbindung $\mathrm{C}_{10} \mathrm{H}_{16} \mathrm{HCl}$ gab. Ferner ein zwischen 205 und $215^{\circ}$ siedendes flüssiges Borneol, die Ameisensäure-, Essigsäure- und Isovaleriansäureester des Borneols and endlich Borneolather, $\left(\mathrm{C}_{10} \mathrm{H}_{17}\right)_{2} \mathrm{O}$, vom Siedepunkt 285 bis $290^{\circ}$.

Diese Körper sind nun teilweise auch im japanischen Baldrianöl neben anderen nachgewiesen worden, wie aus dem folgenden hervorgeht.

Um zunächst eine oberflächliche Trennung der Bestandteile des Öles zu bewirken, wurde ein gröfserer Posten der Destillation mit Wasserdämpfen unterworfen, und das Übergehende in drei Fraktionen aufgefangen.

1 Ber. d. d. chem. Ges. 11, 452.

Arch. d. Pharm. XXVIII. Bds. 9. Heft. 


\section{Dampffraktion $I$.}

Diese Fraktion, welche die niedriger siedenden Anteile des Öles enthielt, wurde durch oft wiederholte Destillation über freiem Feuer, unter Benutzung der Linnemann'schen Dephlegmatorröhre weiter zerlegt. So wurden schliefslich 6 Fraktionen erhalten. 1. Vorlauf bis $155^{0}$ siedend; 2. Siedepunkt 155 bis 1600 ; 3.160 bis $1700 ; 4.170$ bis $180^{\circ}$; 5. 180 bis $200^{\circ}$; 6.200 bis $220^{\circ}$.

Der Vorlauf zeichnete sich durch einen abscheulichen, an faulen Käse erinnernden Geruch aus, der wahrscheinlich von etwas Valeraldehyd herrührte. Er reagierte stark sauer und enthielt grofse Mengen von Essigsäure und Valeriansäure. Ameisensäure war jedoch nicht vorhanden.

Fraktion II, zwischen 155 und $160^{\circ}$ siedend, im Vergleich mit den anderen Fraktionen nur in geringer Menge vorhanden, und von terpentinölartigem Geruch. Mit Amylnitrit, Eisessig und Salzsäure wurde auf die bekannte Weise 1 eine Nitrosochloridverbindung erbalten, deren Schmelzpunkt bei $101^{\circ} \mathrm{lag}$, und die durch alkoholisches Kali leicht in das bei $131^{0}$ schmelzende Nitrosoterpen, welches sich vom Pinen ableitet, übergefuhrt werden konnte. Bei der Untersuchung der Fraktion im Wild'schen Polaristrobometer wurde bei einer Rohrlänge von $100 \mathrm{~mm}$ eine Drehung von $53^{\circ} 5^{\prime}$ nach links beoabachtet. Hiermit ist also die Anwesenheit von Links-Pinen erwiesen.

Bruylants erhielt aus der gleichsiedenden Fraktion des gewöhnlichen Baldrianöles mit Salzsäure ein Monochlorhydrat, $\mathrm{C}_{10} \mathrm{H}_{16} \mathrm{HCl}$. Obgleich der Schmelzpunkt der Verbindung nicht angegeben ist, so ist doch aus dem Siedepunkt des Terpens und der Zusammensetzung des Chlorhydrats der Schlufs berechtigt, dafs hier ebenfalls Pinen vorlag.

Durch Einleiten von trockener Salzsäure in die zwischen 170 und $180^{\circ}$ siedende Fraktion 4 wurde eine bei 49 bis 500 schmelzende Salzsäureverbindung gewonnen. Ein Dichlorhydrat von diesem Schmelzpunkte kann sowohl aus Limonen (Siedepunkt 1750), als auch aus Dipenten (Siedepunkt $180^{\circ}$ ) entstehen. Um nun zu entscheiden, welcher von beiden Kohlenwasserstoffen vorlag, wurde zur Darstellung des Bromids $^{2}$ geschritten und ein solches vom Schmelzpunkt $123^{\circ}$ erhalten. Es lag also Dipenten vor, dessen Bromid bei $125^{0}$ schmilzt, während

1 Wall a ch, Annal. d. Chem. 245, 250.

W alla ch, Annal. d. Chem. 227, 279; 239, 3, 12. 
der Schmelzpunkt des Limonentetrabromids bei 1040 liegt. Es sei jedoch hervorgehoben, dafs die Möglichkeit der Bildung des Dipentens aus Pinen oder Terpineol bei dem während des Fraktionierens massenhaften Auftreten freier Säuren im Vorlauf nicht ganz ausgeschlossen ist.

Die Fraktion 6 von der Siedetemperatur 200 bis 220 enthält Terpineol, $\mathrm{C}_{10} \mathrm{H}_{17} \mathrm{OH}$. Beim Durchschütteln mit einer konzentrierten wässerigen Jodwasserstofflösung entstand die Verbindung $\mathrm{C}_{10} \mathrm{H}_{10} 2 \mathrm{HJ}, 1$ deren Schmelzpunkt nach mehrfachem Umkrystallisieren bei $76^{\circ}$ lag.

Die Fraktion bestand jedoch nicht ausschliefslich aus Terpineol, denn sie drehte bei einer Rohrlänge von $100 \mathrm{~mm}$ die Ebene des polarisierten Lichtstrahls um $15^{\circ} 20^{\prime}$ nach links. Terpineol ist aber optisch inaktiv. 2 Wie sich herausstellte, wurde die optische Aktivitat durch die Anwesenheit von Borneol hervorgebracht. Als nämlich etwas von der Fraktion in einem Uhrglase längere Zeit der Luft ausgesetzt gewesen war, hatte sich ein Teil des Terpineols verflüchtigt, und infolge dessen war Borneol auskrystallisiert. Ob das Borneol nun als solehes ursprünglich in dem Öle vorhanden war, oder ob es sich bei der Destillation durch Zersetzung der Bornylester bildete, wird schwer mit Sicherheit zu entscheiden sein. Für die zweite Auffassung spricht das reichliche Auftreten von Essigsäure und Valeriansäure im Vorlauf.

Die Anwesenheit des Borneols, welches von dem fast gleichsiedenden Terpineol durch fraktionierte Destillation nicht getrennt werden kann, hatte zur Folge, dals, als zur weiteren Identifizierung des Terpineols die Bromierung ausgeführt wurde, wohl ein Bromid in reichlicher Menge entstand, dessen Schmelzpunkt jedoch sich selbst nach häufigem Um. krystallisieren inkonstant zeigte und immer beträchtlich unter dem des zu Terpineol gehörigen Dipententetrabromids lag.

Durch Behandeln mit trockener Salzsäure wurde kein festes Chlorhydrat erhalten. Als das Reaktionsprodukt längere Zeit offen an der Luft gestanden hatte, trat der Geruch nach Borneol auf, welches dann nach einiger Zeit auskrystallisierte. Es wurde durch Aufstreichen auf poröse Thonteller von der anhaftenden Flüssigkeit befreit, aus Petroläther umkrystallisiert und durch Bestimmung seines Schmelzpunktes mit Sicherheit als Borneol erkannt.

Trotzdem die für Terpineol charakteristischen Verbindungen $\mathrm{C}_{10} \mathrm{H}_{16} \mathrm{Br}_{\mathbf{4}}$

1 Walla ch, Annal. d. Chem. 230, 265.

2 Wallach, Annal. d. Chem. 239, 21. 
und $\mathrm{C}_{10} \mathrm{H}_{16} 2 \mathrm{HCl}$ aus den angefuhrten Gründen nicht in reinem $\mathrm{Zu}$ stande dargestellt werden konnten, kann doch aus dem leichten Entstehen des Körpers $\mathrm{C}_{10} \mathrm{H}_{16} 2 \mathrm{JH}$ das Vorhandensein des Terpineols als bewiesen angesehen werden.

Utber die Zusammensetzung der zwischen 205 und $215^{\circ}$ siedenden Anteile des gewöhnlichen Baldrianöles giebt Bruylants an, dafs dieselben aus einem flüssigen Borneol $\mathrm{C}_{10} \mathrm{H}_{18} \mathrm{O}$ beständen.

Es liegt nun nahe, anzunehmen, dafs er ebenfalls ein Gemenge von Terpineol (Siedep. 215-2180) und Borneol (Siedep. 2120) unter Händen hatte, denn er erhielt bei der Oxydation Kampfer, ferner Ameisensäure, Essigsäure und Valeriansäure. Der Kampfer hätte sich demnach aus dem Borneol gebildet, während die Entstehung der anderen Säuren aus dem Terpineol zu erk]ären wäre.

\section{Dampffraktion II.}

Die Fraktion siedete zwischen 220 und 2900 . Zunächst wurde das zwischen 220 und $260^{\circ}$ Übergehende mit Hilfe des Linnemannschen Aufsatzes heraus fraktioniert und in beträchtlicher Menge erbalten. Das Destillat wurde nun der Einwirkung von alkoholischem Kali auf dem Wasserbade ansgesetzt. Nach einigen Stunden war die Verseifung vollendet. Nachdem nach dem Verdünnen mit Wasser der Alkohol verdunstet und die wässerige Schicht von der öligen durch den Scheidetrichter getrennt worden war, wurde letztere der Destillation unterworfen; sie erstarrte mit Ausnahme der zuletzt übergehenden Anteile fast vollständig in der Vorlage. Die feste Masse, durch Umkrystallisieren aus Petroläther in grofsen perlmutterglänzenden Tafeln erhalten, bestand ausschliefslich aus Borneol, und zwar aus der linksdrehenden Modifikation. Die wässerige Kalilösung bestand aus den Salzen der Essig- und Isovaleriansäure, die beim Ansäuren mit Schwefelsäure in Freiheit gesetzt wurden. Das zwischen 220 und $260^{\circ}$ Siedende des Kessoöls besteht also aus den Essigsäure- und Isovaleriansäureestern des Borneols, welche nach Bruylants neben dem Ameisensäureester des Borneols auch im Baldrianöl vorhanden sind. Die letztgenannte Verbindung fehlt im Kessoöl jedoch ganz.

Das oberhalb $260^{\circ}$ Siedende mufste, um eine allzugrolse Überhitzung zu vermeiden, ohne Linnemann'schen Aufsatz fraktioniert werden. Zwischen 260 und $280^{\circ}$ ging eine farblose Flüssigkeit iiber, die den ausgesprochenen charakteristischen Sesquiterpengeruch zeigte. 
Versuche, ein Chlorhydrat aus derselben darzustellen, führten zu keinem Resultate.

\section{Dampf fraktion III.}

Während bei der Destillation mit Wasserdämpfen der gröfsere Teil des Destillats leichter als Wasser war - Dampffraktion I und II sammelte sich gegen Ende der Destillation ein immerhin sehr beträchtlicher Teil auf dem Boden der Vorlage an, war also schwerer als Wasser, eine Erscheinung, die bei der Destillation der deutschen Baldrianwurzel niemals beobachtet worden war. Das dunkelgrün gefärbte Destillat siedete der Hauptmenge nach nahe um 300\%. Da wir in demselben Ester höherer Fettsäuren des Borneols vermuteten, so verseiften wir $500 \mathrm{~g}$ desselben mit $100 \mathrm{~g} \mathrm{KOH}$, die in $200 \mathrm{~g}$ Alkohol gelöst waren. Durch Zusatz von Wasser schied sich ein dickes Öl $a b$, welches beim Destillieren zwischen 290 und $305^{\circ}$ überging. Das Destillat erstarrte gröfstenteils in der Vorlage und wurde durch Absaugen von einer grünblau gefärbten Flüssigkeit getrennt, welche möglicherweise identisch ist mit den gleichgefärbten und um dieselbe Temperatur siedenden Anteilen des Kamillenöles, des Achilleaöles, Kampferöles und anderer ätherischer Öle. 1

Die Ausbeute an festem Verseifungsprodukt, welches jedoch kein Borneol war, wie wir erwartet hatten, betrug etwa ein Drittel des angewandten Öles.

Um zunächst die Natur des sauren Komponenten des verseiften Körpers festzustellen, wurde die wässerige alkalische Flüssigkeit nach dem fraktionierten Ansäuern mit Schwefelsäure der Destillation mit Wasserdämpfen unterworfen, und auf diese Weise drei verschiedene Destillate erhalten, welche mit Natriumcarbonat neutralisiert und darauf mit Silbernitratlösung versetzt wurden. Die ausgefallenen Silbersalze gaben nach dem Umkrystallisieren bei der Analyse folgende, auf essigsaures Silber stimmende Zahlcn:

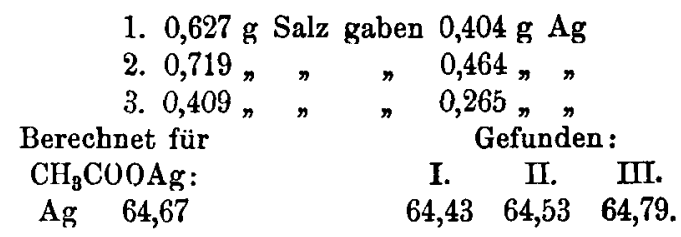

${ }^{1}$ Im Baldrianöl kommt nach Flü ckiger ebenfalls ein blaugefärbtes Öl, das über $300^{\circ}$ siedet, vor (cfr. Archiv d. Pharm. 209, 1876, 204). 


\section{Kessylalkohol.}

Das oben erwähnte, nach der Destillation in der Vorlage als fester Körper erhaltene Verseifungsprodukt wurde zur Reinigung mehrmals aus 70 proz. Alkohol umkrystallisiert und in schönen durchsichtigen Krystallen erhalten. Wir nennen diesen Körper, der sich seiner chemischen Natur nach als Alkohol herausstellte, Kessylalkohol. Die Elementaranalyse der uber Schwefelsäure getrockneten Substanz ergab folgendes:

1. $0,2259 \mathrm{~g}$ Substanz gaben $0,2218 \mathrm{H}_{2} \mathrm{O}=0,02464 \mathrm{H}$ und $0,6211 \mathrm{CO}_{2}$ $=0,16939 \mathrm{C}$.

2. $0,2069 \mathrm{~g}$ Substanz gaben $0,2012 \mathrm{H}_{2} \mathrm{O}=0,022354 \mathrm{H}$ und $0,5678 \mathrm{CO}_{2}$ $=0,15485 \mathrm{C}$.

\begin{tabular}{cccc} 
& \multicolumn{2}{c}{ Gefunden: } & Berechnet für \\
& I. & II. & $\left(\mathrm{C}_{7} \mathrm{H}_{12} \mathrm{O}\right) \mathrm{x}$ \\
C & 74,98 & 74,84 & 75,0 \\
H & 10,90 & 10,80 & $10,71$.
\end{tabular}

Die Bestimmung des Molekulargewichts wurde nach der Raoultschen Methode in dem von Beckmann 1 empfohlenen Apparat ausgefiihrt. Als Lösungsmittel diente Benzol.

\begin{tabular}{|c|c|c|c|}
\hline & & \multicolumn{2}{|c|}{ Molekulargewicht } \\
\hline $\begin{array}{l}\text { Prozentgebalt } \\
\text { d.Lösung: }\end{array}$ & $\begin{array}{l}\text { Beobachtete } \\
\text { Erniedrigung : }\end{array}$ & Gefunden: & $\begin{array}{l}\text { Berechnet für: } \\
\mathrm{C}_{\mathbf{1 4}} \mathrm{H}_{24} \mathrm{O}_{2}\end{array}$ \\
\hline & 0,300 & 219 & 224 \\
\hline 2,209 & 0,465 & 232 & - \\
\hline
\end{tabular}

Hiernach kommt also dem Kessylalkohol die Formel $\mathrm{C}_{14} \mathrm{H}_{24} \mathrm{O}_{2}$ zu. Der Kessylalkohol ist geruchlos, unlöslich in Wasser, leicht löslich in Alkohol, Äther, Chloroform, Benzol und Petroläther. Er schmilzt bei $85^{\circ}$ und siedet bei einem Druck von $11 \mathrm{~mm}$ zwischen 155 und 1560 , bei gewöhnlichem Luftdruck ganz unzersetzt bei $300-3020$. Die Lösungen des Kessylalkohols sind optisch aktiv, und zwar wurde für eine 10 proz. alkoholische Lösung bei einer Rohrlänge von $100 \mathrm{~mm}$ eine Ablenkung des polarisierten Lichtstrahls um $30 \quad 39^{\prime}$ nach links beobachtet.

Ausgezeichnet ist der Alkohol durch sein grofses Krystallisationsvermögen. Er ist aus Alkohol und $\ddot{A}$ ther leicht in sehr gut ausgebildeten Krystallen zu erhalten.

1 Beckmann, Zeitschr. f. physikal Them. $\Pi, 638$. 
Herr Dr. K. Bufz, Assistent am mineralogischen Institut der Universität Bonn, welcher die Freundlichkeit hatte, die krystallographischen Bestimmungen auszuführen, teilt uns hiertiber folgendes mit:

Die Substanz krystallisiert im rhombischen System. Die Ebene der optischen Axen liegt senkrecht zur Vertikalaxe. Die spitze Bissectrix fällt mit der Axe a zusammen. Der scheinbare Winkel der optischen Axen beträgt ungefähr $65^{\circ}$.

Die auftretenden Formen sind (siehe Figur):

$$
\begin{array}{rlrl}
\mathrm{a}= & \infty \mathrm{P} \infty(100) & \mathrm{o}=\mathrm{P} \overline{ }(101) \\
\mathrm{m}= & \infty \mathrm{P}(110) & \mathrm{s}=2 \mathrm{P} \infty(102) \\
\mathrm{p}= & \infty \mathrm{P} \overline{\mathrm{a}}(120) & & \\
& \mathrm{a}: \mathrm{b}: \mathrm{c}=0,9936: 1: 0,4369 .
\end{array}
$$

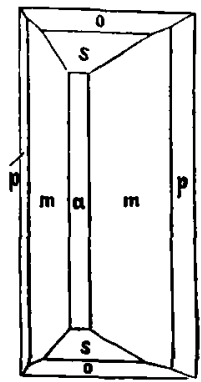

Die Krystalle, bis zu $1 \mathrm{~cm}$ grofs, sind in der Richtung der Vertikalaxe verlängert. Vorherrschend ausgebildet ist das Prisma $\infty \mathrm{P}$, während $\infty \mathrm{P} \bar{\infty}$ und $\infty \mathrm{P} \overline{\mathrm{a}}$ weniger grols auftreten und bei vielen Krystallen uberhaupt nicht vorhanden sind. Die Domen sind an allen Krystallen ungefähr gleichnälsig ausgebildet. Die Flächen sind zwar glänzend, aber nicht glatt und geben daher keine guten Bilder.

Die Messungen ergaben:

Gemessen: Berechnet:

$$
\begin{array}{lc}
110: \overline{11} 0=90^{\circ} 22^{\prime} & - \\
100: 101=66^{\circ} 16^{\prime} & - \\
101: \overline{101}=47^{\circ} 25^{\prime} & 47^{\circ} 28^{\prime} \\
101: 102=17^{\circ} 31^{\prime} & 17^{\circ} 36^{\prime} \\
102: 100=48^{\circ} 51^{\prime} & 48^{\circ} 40^{\prime} \\
102: 102=82^{\circ} 27^{\prime} & 82^{\circ} 40^{\prime} \\
100: 110=44^{\circ} 44^{\prime} & 44^{\circ} 49^{\prime} \\
120: 1 \overline{20}=53^{\circ} 42^{\prime} & 53^{\circ} 26^{\prime} \\
120: 100=63^{\circ} 12^{\prime} & 63^{\circ} 17^{\prime} \\
120: 110=18^{\circ} 28^{\prime} & 18^{\circ} 28^{\prime}
\end{array}
$$

$\beta=1,5244(\mathrm{Na})$. Dispersion sehr schwach: $\rho<\mathrm{v}$. Doppelbrechung negativ.

Bringt man Kessylalkohol entweder in trockenem Äther gelöst oder in Substanz mit Acetylchlorid oder Phosphorpentachlorid zusammen, so erwärmt sich das Gemisch unter Entweichen von Salzsăuredämpfen. Hierbei färbt sich die Lösung prachtvoll dunkelkirschrot.

Zur Darstellung der Acetylverbindung schien jedoch Essigsäureanhydrid geeigneter als Acetylchlorid, da die Salzsäure, wie die Färbung zeigte, Anlals zur Bildung von unerwünschten Nebenprodukten gab. 


\section{Kess ylacetat.}

Beim Kochen von Kessylalkohol mit überschüssigem Essigsäureanhydrid, unter Zusatz von etwas wasserfreiem Natriumacetat erhält man nach dem Neutralisieren mit Alkalien ein dickflüssiges Öl, welches bei einem Druck von $15-16 \mathrm{~mm}$ zwischen 178 und 1790 , bei gewöhnlichem Luftdruck nicht ganz unzersetzt gegen $300^{\circ}$ siedet.

Die Elementaranalyse ergab auf die Formel $\mathrm{C}_{\mathbf{1 4}} \mathrm{H}_{23} \mathrm{O}_{2}-\mathrm{CH}_{3} \mathrm{CO}$ stimmende Zahlen.

$0,2200 \mathrm{~g}$ Substanz gaben $0,1996 \mathrm{H}_{2} \mathrm{O}=0,02217 \mathrm{II}$ und $0,5791 \mathrm{CO}_{2}$ $=0,15793 \mathrm{C}$.

$\begin{array}{cc}\text { Gefunden: } & \text { Berechnet für } \mathrm{C}_{16} \mathrm{H}_{26} \mathrm{O}_{3}: \\ \text { C } 71,79 & 72,18 \text { Proz. } \\ \text { H } 10,08 & 9,77\end{array}$

In Übereinstimmung mit der angeführten Formel wurde durch Verseifen mit Kalilauge und Zurïcktitrieren des Überschusses gefunden, dafs nur eine Acetylgrupge eingetreten war, dafs also der Kessylalkohol als einsäuriger Alkohol anzusehen ist. Der durch Verseifen des Acetats wiedergewonnene Kessylalkohol zeigte denselben Schmelzpunkt wie das Ausgangsmaterial.

Eine in Benzol ausgefuhrte Molekulargewichtsbestimmung bestätigte ebenfalls die Formel $\mathrm{C}_{14} \mathrm{H}_{23} \mathrm{O}_{2} \mathrm{CH}_{3} \mathrm{CO}$.

\begin{tabular}{cccc} 
Prozentgehalt & Beobachtete & \multicolumn{2}{c}{ Molekulargewicht } \\
der Lösung: & Erniedrigung: & Gefunden: & Berechnet: \\
0,3113 & $0,58^{\circ}$ & 262 & 266 \\
1,0039 & $1,89^{\circ}$ & 260 &
\end{tabular}

Das Kessylacetat stellt ein dickfiüssiges farbloses Öl von sehr schwachem Geruch dar, welches bei $-20^{0}$ nicht fest wird. Der Körper ist unlöslich in Wasser, leicht hingegen in Äther, Alkohol, Chloroform und Petroläther. Mit Säuren giebt er, wie der Alkohol, intensive rote bis violette Färbungen.

Der polarisierte Lichtstrahl wird durch das Kessylacetat bei einer Röhrenlänge von $100 \mathrm{~mm}$ um $70^{\circ} 6^{\prime}$ nach links abgelenkt.

Bei der Oxydation mit Kaliumpermanganat wurden nur flüssige Produkte, niedere Fettsäuren, erhalten. Ein gleiches Resultat hatten Oxydationsversuche mit Kaliumdichromat und Schwefelsäure.

Das Kessylacetat kann auch direkt durch fraktionierte Destillation aus dem Kessoöl gewonnen werden. Auf diese Weise wurde eine ziemlich konstant bei $15 \mathrm{~mm}$ Druck um $178^{0}$ siedende Flüssigkeit ge- 
wonnen. Rein war dieses Kessylacetat jedoch nicht, sondern etwas grün gefärbt, auch betrug der Drehungswinkel nur -480 , ein Beweis, dafs noch Verunreinigungen zugegen waren.

\section{Oxydation des Kessylalkohols.}

In der Hoffnung, durch Oxydation des Kessylalkohols zu einfacheren Körpern zu gelangen, welche Schlüsse auf seine Konstitution zu ziehen erlaubten, wurden verschiedene Versuche ausgeführt. Eine kalt gesättigte Lösung von Kaliumpermanganat verbrannte einen Teil des Alkohols vollständig zu Kohlensäure, welche in grofsen Mengen entwich, und liefs einen Teil völlig unangegriffen. Die Oxydation mit Salpetersäure hatte ebenfalls keinen Erfolg.

Weniger heftig wirkt die Lösung von Kaliumdichromat, der man nur die Hälfte der theoretisch notwendigen Menge Schwefelsäure zusetzt.

Es wurden so $20 \mathrm{~g}$ Kessylalkohol mit $24 \mathrm{~g}$ Kaliumdichromat, $120 \mathrm{~g}$ Wasser und $20 \mathrm{~g}$ Schwefelsäure mehrere Stunden lang auf dem Wasserbade erhitzt. Nach dem Erkalten wurde die auf der wässerigen Flüssigkeit erstarrte Kruste zerrieben, mit kaltem Wasser ausgewaschen und aus 70 proz. Alkohol umkrystallisiert. Aus diesem Lösungsmittel wurde das Oxydationsprodukt in derben, bei 104 bis $105^{\circ}$ schmelzenden Nadeln erhalten.

Der Körper siedet bei einem Druck von $11 \mathrm{~mm}$ zwischen 162 bis 1630 und zwischen 305 bis 3070 bei gewöhnlichem Luftdruck. Die Ebene des polarisierten Lichts wird durch eine 10 proz. alkoholische Lösung bei $100 \mathrm{~mm}$ Rohrlänge um $1806^{\prime}$ nach rechts abgelenkt. Bemerkenswert ist, dafs bei der Oxydation eine Umkehr der Drehungsrichtung stattgefunden hat.

Bei der Elementaranalyse wurden folgende Zahlen erhalten:

$$
\begin{array}{cc}
0,2145 \mathrm{~g} \text { Substanz } & \text { gaben } \\
=0,1961 \mathrm{H}_{2} \mathrm{O}=0,02178 \mathrm{H} \text { und } 0,5962 \mathrm{CO}_{2} \\
\text { Gefunden: } & \text { Berechnet für } \mathrm{C}_{14} \mathrm{H}_{22} \mathrm{O}_{2}: \\
\text { C } 75,80 & 75,67 \mathrm{Proz} \\
\mathrm{H} 10,15 & 9,90
\end{array}
$$

Die Molekulargewichtsbestimmung ergab auf die Formel $\mathrm{C}_{14} \mathrm{H}_{\mathbf{2}} \mathrm{O}_{\mathbf{2}}$ gutstimmende Resultate.

\begin{tabular}{cccc} 
Prozentgehalt & Beobachtete & \multicolumn{2}{c}{ Molekulargewicht } \\
der Lösung: & Erniedrigung: & Gefunden : & Berechnet: \\
0,8243 & $0,187^{\circ}$ & 216 & 222 \\
2,158 & $0,475^{\circ}$ & 222 &
\end{tabular}


Da demnach das Oxydationsprodukt um 2 H-Atome ärmer ist als der Alkohol, so waren wir geneigt, letzteren für einen sekundären Alkohol, ersteres für das dazu gehörige Keton anzusprechen. Leider sind jedoch die Versuche zur Feststellung der Natur des Oxydationsprodukts ohne Erfolg geblieben. Weder mit Phenylhydrazin noch mit Hydroxylamin wurden greifbare Verbindungen erhalten. Da es uns nicht unwahrscheinlich schien, dafs die Beziehungen des Alkohols zu dem Oxydationsprodukt ähnlicher Art wären, wie die des Borneols zum Kampfer, oder des Menthols zum Menthon, so wurde versucht, durch metallisches Natrium das Oxydationsprodukt wieder in den Alkohol tiberzufuhren. Jedoch auch dies milslang, es blieb schlielslich nur ein dickes, unerfreuliches Öl übrig.

Der Körper zeigte sich überhaupt sehr indifferent gegen sehr viele Agentien. Oxydationsmittel verbrannten ihn zu Kohlensäure und niederen Fettsäuren. Mit wasserabspaltenden Mitteln, wie Kaliumdisulfat und Schwefelsäure, wurden nur zähflüssige Öle erhalten, die keinen konstanten Siedepunkt zeigten und welche keineswegs zu weiteren Untersuchungen einluden.

Wir hoffen jedoch in späterer Zeit in der Lage zu sein, Aufschlüsse über die Beziehungen des Oxydationsprodukts zum Alkohol sowie uber die die Konstitution beider geben zu können.

Das Kessoöl setzt sich also aus folgenden Bestandteilen zusammen:

1. Aldehyde und niedere Fettsäuren.

2. Linkspinen.

3. Dipenten.

4. Terpineol.

5. Linksborneol.

6. Essigsäurebornyläther.

7. Isovaleriansäurebornyläther.

8. Ein sesquiterpenartiger Körper.

9. Kessylacetat $\mathrm{C}_{14} \mathrm{H}_{23} \mathrm{O}_{2} \mathrm{CH}_{3} \mathrm{CO}$.

10. Ein blaues Öl von noch unbekannter Zusammensetzung.

Leipzig.

Laboratorium der Fabrik ätherischer Öle von Schimmel \& Cı. 